\title{
SCALAR CURVATURES OF HERMITIAN METRICS ON THE MODULI SPACE OF RIEMANN SURFACES
}

\author{
YUNHUI WU
}

\begin{abstract}
In this article we show that any finite cover of the moduli space of closed Riemann surfaces of $g$ genus with $g \geqslant 2$ does not admit any complete finite-volume Hermitian metric of non-negative scalar curvature.

Moreover, we also show that the total mass of the scalar curvature of any almost Hermitian metric, which is equivalent to the Teichmüller metric, on any finite cover of the moduli space is negative provided that the scalar curvature is bounded from below.
\end{abstract}

\section{INTRODUCTION}

Let $S_{g}$ be a closed surface of genus $g$ with $g \geqslant 2, \operatorname{Mod}\left(S_{g}\right)$ be the mapping class group and $T_{g}$ be the Teichmüller space of $S_{g}$. Topologically $T_{g}$ is a complex manifold of complex dimension $3 g-3$, which carries various $\operatorname{Mod}\left(S_{g}\right)$-invariant metrics which descend into metrics on the moduli space $\mathbb{M}_{g}$ of $S_{g}$ with respective properties. For examples, the Teichmüller metric, Kobayashi metric and Caratheódory metric are complete and Finsler. The Weil-Petersson metric is Kähler [1, incomplete [4, 17] and has negative sectional curvatures [18]. The Asymptotic Poincaré metric, Induced Bergman metric, Kähler-Einstein metric, McMullen metric, Ricci metric, and perturbed Ricci metric are complete and Kähler. In [10, 11, 13, 21, the authors showed that the metrics listed above except the Weil-Petersson metric are equivalent.

It is shown that the perturbed Ricci metric [10, 11] has pinched negative Ricci curvature. So does the scalar curvature of the perturbed Ricci metric. And the McMullen metric [13] has negative scalar curvature at certain points since the metric, restricted on certain thick part of the moduli space, is the Weil-Petersson metric. However, in [7] Farb and Weinberger show that any finite cover $M$ of the moduli space $\mathbb{M}_{g}(g \geqslant 2)$ admits a complete finitevolume Riemannian metric of (uniformly bounded) positive scalar curvature. They also show that this metric is not quasi-isometric to the Teichmüller metric. And they conjecture (see Conjecture 4.6 in [5])

Conjecture 1.1 (Farb-Weinberger). Let $S_{g}$ be a surface of $g$ genus with $g \geqslant 2$. Then any finite cover $M$ of the moduli space $\mathbb{M}_{g}$ of $S_{g}$ does not

1991 Mathematics Subject Classification. 30F60, 32G15.

Key words and phrases. Moduli space, Scalar curvature, Teichmüller metric. 
admit a finite volume Riemannian metric of (uniformly bounded) positive scalar curvature in the quasi-isometry class of the Teichmüller metric.

Let $M$ be any finite cover of $\mathbb{M}_{g}$. The natural complex structure on the Teichmüller space descends into a complex structure on $M$. In this paper we will focus complete Hermitian metrics on $M$ with this complex structure. Our first result is

Theorem 1.2. Let $S_{g}$ be a surface of $g$ genus with $g \geqslant 2$ and $M$ be a finite cover of the moduli space $\mathbb{M}_{g}$ of $S_{g}$. Then for any complete finite-volume Hermitian metric $\|\cdot\|$ on $M$, the scalar curvature Sca of $(M,\|\cdot\|)$ satisfies

$$
\inf _{p \in M} \operatorname{Sca}(p)<0 .
$$

As introduced before there is a list of canonical metrics which are equivalent (or quasi-isometric) to the Teichmüller metric $\|\cdot\|_{T}$ (see [10, 11, 13, 21]). Our second aim is the following uniform upper bound on the infimum of the scalar curvature of any Hermitian metric on a given class.

Theorem 1.3. Let $S_{g}$ be a surface of $g$ genus with $g \geqslant 2$ and $M$ be a finite cover of the moduli space $\mathbb{M}_{g}$ of $S_{g}$. Given two constants $k_{1}, k_{2}>0$, then for any Hermitian metric $\|\cdot\|$ on $M$ with $k_{1}\|\cdot\| \leqslant\|\cdot\|_{T} \leqslant k_{2}\|\cdot\|$, there exists a constant $K\left(k_{1}, g\right)>0$ only depending on $k_{1}$ and $g$ such that the scalar curvature satisfies

$$
\inf _{p \in(M,\|\cdot\|)} \operatorname{Sca}(p) \leqslant-K\left(k_{1}, g\right)<0 .
$$

Theorem 1.3 confirms Conjecture 1.1 for the Hermitian case. For the general Riemannian case, we have been told by Prof. Farb in [6] that Conjecture 1.1 is confirmed by him and Weinberger in a forthcoming paper. And their method is completely different from the method in this article.

Both Theorem 1.2 and Theorem 1.3 tell that the scalar curvature of any Hermitian metric, which is equivalent to the Teichmüller metric, on the moduli space is negative at certain points. It is natural to ask whether the total mass of the scalar curvature could be positive. Our last result tells that this is impossible if we assume the metric is almost Hermitian and its scalar curvature has a low bound.

Theorem 1.4. Let $S_{g}$ be a surface of $g$ genus with $g \geqslant 2$ and $M$ be a finite cover of the moduli space $\mathbb{M}_{g}$ of $S_{g}$. Let $\| \cdot \mid$ be an almost Hermitian metric on $M$ satisfying $\|\cdot\| \approx\|\cdot\|_{T}$. Assume that the scalar curvature of $(M,\|\cdot\|)$ is bounded from below, then the total mass of the scalar curvature satisfies

$$
\int_{p \in(M,\|\cdot\|)} \operatorname{Sca}(p) \mathrm{dVol}(p)<0 .
$$

Theorem 1.4 applies to the Asymptotic Poincaré metric, Induced Bergman metric, McMullen metric and Ricci metric, which is new. Actually it also applies to any metric in the the convex hull of the Kähler-Einstein metric, 
perturbed Ricci metric and the four metrics above.

It is interesting to know whether there exists a Hermitian metric $\|\cdot\|$ on a finite cover $M$ of $\mathbb{M}_{g}$ such that $\|\cdot\|$ is equivalent to the Teichmüller metric and $(M,\|\cdot\|)$ has non-negative scalar curvature outside some compact subset of $M$. We hope the method in this paper is helpful for this question.

1.1. Plan of the paper. In section 2 we review some recent developments on the canonical metrics on the moduli space of surfaces and recall one formula of S. S. Chern which is crucial for this article. In section 3 we establish Theorem 1.2. Theorem 1.3 is proved in section 4. And we will finish the proof of Theorem 1.4 in section 5 .

\section{Notations and Preliminaries}

2.1. Surfaces. Let $S_{g}$ be a closed surface of $g$ genus with $g \geqslant 2$, and $M_{-1}$ denote the space of Riemannian metrics with constant curvature -1 , and $X=\left(S_{g}, \sigma|d z|^{2}\right)$ be an element in $M_{-1}$. The group Diff 0 of diffeomorphisms of $S_{g}$ isotopic to the identity, acts by pull-back on $M_{-1}$. The Teichmüller space $T_{g}$ of $S_{g}$ is defined by the quotient space

$$
T_{g}=M_{-1} / \operatorname{Diff}_{0} \text {. }
$$

Let Diff + be the group of orientation-preserving diffeomorphisms of $S_{g}$. The mapping class group $\operatorname{Mod}\left(S_{g}\right)$ is defined as

$$
\operatorname{Mod}\left(S_{g}\right)=\operatorname{Diff}_{+} / \operatorname{Diff}_{0} .
$$

The moduli space $\mathbb{M}_{g}$ of $S_{g}$ is defined by the quotient space

$$
\mathbb{M}_{g}=T_{g} / \operatorname{Mod}\left(S_{g}\right) \text {. }
$$

The Teichmüller space has a natural complex structure, and its holomorphic cotangent space $T_{X}^{*} T_{g}$ is identified with the quadratic differentials

$$
Q D(X)=\left\{\phi(z) d z^{2}\right\}
$$

while its holomorphic tangent space is identified with the harmonic Beltrami differentials

$$
H B D(X)=\left\{\frac{\overline{\phi(z)}}{\sigma(z)} \frac{d \bar{z}}{d z}\right\}
$$

Recall that the Teichmüller metric $\|\cdot\|_{T}$ on $T_{g}$ is defined as

$$
\left\|\frac{\overline{\phi(z)}}{\sigma(z)}\right\|_{T}:=\sup _{\psi d z^{2} \in Q D(X), \quad \int_{X}|\psi|=1} \operatorname{Re} \int_{X} \frac{\overline{\phi(z)}}{\sigma(z)} \cdot \psi(z) \frac{d z \wedge d \bar{z}}{-2 \mathbf{i}} .
$$

The Weil-Petersson metric $\|\cdot\|_{W P}$ is the Hermitian metric on $T_{g}$ arising from the the Petersson scalar product

$$
<\varphi, \psi>=\int_{X} \frac{\varphi(z) \cdot \overline{\psi(z)}}{\sigma(z)} \frac{d z \wedge d \bar{z}}{-2 \mathbf{i}}
$$

via duality. 
Both the Teichmüller metric and the Weil-Petersson metric are $\operatorname{Mod}\left(S_{g}\right)$ invariant.

Let $\|\cdot\|_{1}$ and $\|\cdot\|_{2}$ be any two metrics on $T_{g}$. We call $\|\cdot\|_{1}$ is controlled above by $\|\cdot\|_{2}$ if there exists a positive constant $k$ such that

$$
\|\cdot\|_{1} \leqslant k\|\cdot\|_{2}
$$

which is denoted by $\|\cdot\|_{1} \prec\|\cdot\|_{2}$.

The Cauchy-Schwarz inequality and the Gauss-Bonnet formula tell us that

$$
\|\cdot\|_{W P} \prec\|\cdot\|_{T} \cdot
$$
if

We call the two metrics $\|\cdot\|_{1}$ and $\|\cdot\|_{2}$ are equivalent (or quasi-isometric)

$$
\|\cdot\|_{1} \prec\|\cdot\|_{2} \quad \text { and } \quad\|\cdot\|_{2} \prec\|\cdot\|_{1} \text {. }
$$

We denote it by $\|\cdot\|_{1} \approx\|\cdot\|_{2}$.

It is not hard to see that $\|\cdot\|_{W P}$ is not equivalent to $\|\cdot\|_{T}$ because the Weil-Petersson metric is incomplete and the Teichmüller metric is complete.

2.2. Kähler metrics on $\mathbb{M}_{g}$. In this subsection we briefly review some properties of the following three Kähler metrics $\mathbb{M}_{g}$ : the McMullen metric, the Ricci metric, and the perturbed Ricci metric. They will be applied to prove Theorem 1.3 in section 4 .

2.2.1. McMullen metric. In [13] McMullen constructed a new metric $\|\cdot\|_{M}$ on $\mathbb{M}_{g}$, called the McMullen metric. More precisely, let Log: $\mathbb{R}_{+} \rightarrow[0, \infty)$ be a smooth function such that

(1). $\log (x)=\log (x)$ if $x \geqslant 2$;

(2). $\log (x)=0$ if $x \leqslant 1$.

For suitable choices of small constants $\epsilon, \delta>0$, the Kähler form of the McMullen metric is

$$
\omega_{M}=\omega_{W P}-\mathbf{i} \delta \sum_{\ell_{\gamma}(X)<\epsilon} \partial \bar{\partial} \log \frac{\epsilon}{\ell_{\gamma}}
$$

where the sum is taken over primitive short geodesics $\gamma$ on $X$. Restricted on certain thick part of $\mathbb{M}_{g}$ the McMullen metric is exactly the Weil-Petersson metric. McMullen in [13] proved that this metric is Kähler hyperbolic and has bounded geometry. He also showed that

Theorem 2.1 (McMullen). On the moduli space $\mathbb{M}_{g},\|\cdot\|_{M} \approx\|\cdot\|_{T}$. 
2.2.2. Ricci metric and perturbed Ricci metric. In [15, 18] it is shown that the Weil-Petersson metric has negative sectional curvature. The negative Ricci curvature tensor defines a new metric $\|\cdot\|_{\tau}$ which is called the Ricci metric on $\mathbb{M}_{g}$. Trapani in [16] proved $\|\cdot\|_{\tau}$ is a complete Kähler metric.

In [10] Liu-Sun-Yau perturbed the Ricci metric along the Weil-Petersson direction to give new metrics on $\mathbb{M}_{g}$ which are called the perturbed Ricci metrics denoted by $\|\cdot\|_{L S Y}$. More precisely, let $\omega_{\tau}$ be the Kähler form of the Ricci metric, for any constant $C>0$, the Kähler form of the perturbed Ricci metric is

$$
\omega_{L S Y}=\omega_{\tau}+C \cdot \omega_{W P}
$$

In [10] the authors showed that both $\|\cdot\|_{\tau}$ and $\|\cdot\|_{L S Y}$ have bounded geometry. By using Yau's generalized Schwarz Lemma [20] they also showed that

Theorem 2.2 (Liu-Sun-Yau). On the moduli space $\mathbb{M}_{g}$,

$$
\|\cdot\|_{L S Y} \approx\|\cdot\|_{\tau} \approx\|\cdot\|_{M} .
$$

Furthermore, in one of their subsequent papers [1] they showed that

Theorem 2.3 (Liu-Sun-Yau). With a suitable choice of the constant $C$, there exists two positive numbers $C_{1}, C_{2}$ such that the Ricci curvature of the perturbed Ricci metric $\|\cdot\|_{L S Y}$ satisfies

$$
-C_{1} \leqslant R i c_{\|\cdot\|_{L S Y}} \leqslant-C_{2}<0 .
$$

Moreover, they also showed in [1] that the perturbed Ricci metric $\|\cdot\|_{L S Y}$ has negatively pinched holomorphic sectional curvatures, which is known to be the first complete metric on the moduli space with this property. And they use this property and the Schwarz-Yau lemma to prove a list of canonical metrics on the moduli space are equivalent.

2.3. The ratio of intermediate volume elements. In this subsection we briefly review a formula of Chern in [3] which is crucial for this article. For the notations and computations one can also refer to [12 for more details.

Let $M$ and $N$ be two $2 n$-dimensional Hermitian manifolds and $f: M \rightarrow$ $N$ be a holomorphic mapping. Let $\left\{\theta_{i}\right\}_{1 \leqslant i \leqslant n},\left\{\omega_{\alpha}\right\}_{1 \leqslant \alpha \leqslant n}$ be the unitary coframe fields of $M$ and $N$ respectively. There exists complex numbers $a_{\alpha i}$ such that

$$
f^{*} \omega_{\alpha}=\sum_{i=1}^{n} a_{\alpha i} \theta_{i}
$$

Then

$$
f^{*}\left(\omega_{\alpha} \wedge \bar{\omega}_{\alpha}\right)=\sum_{i=1}^{n} \sum_{j=1}^{n} a_{\alpha i} \bar{a}_{\alpha j} \theta_{i} \wedge \bar{\theta}_{j}
$$


By raising equation (2.2) to the $n^{\text {th }}$ power, the ratio of intermediate volume elements $v$ is defined as

$$
v:=\frac{f^{*}\left(\sum_{\alpha=1}^{n} \omega_{\alpha} \wedge \bar{\omega}_{\alpha}\right)^{n}}{\left(\sum_{i=1}^{n} \theta_{i} \wedge \bar{\theta}_{i}\right)^{n}} .
$$

Linear algebra gives that

$$
v=\frac{f^{*}\left(\mathrm{dVol}_{N}\right)}{\mathrm{dVol}_{M}}=D \cdot \bar{D}
$$

where

$$
D=\operatorname{det}\left(a_{\alpha \beta}\right) .
$$

Let $\Delta$ be the Laplace operator of $M$. Now we are ready to state Chern's formula.

Theorem 2.4 (Chern).

$$
\frac{\Delta v}{4}=\sum_{k=1}^{n} D_{k} \cdot \overline{D_{k}}+\frac{v}{2}\left(\mathrm{Sca}-\sum_{1 \leqslant \alpha, \beta, k \leqslant n} a_{\alpha k} \bar{a}_{\beta k} \widetilde{\operatorname{Ric}}_{a \bar{\beta}}\right)
$$

where $\sum_{k=1}^{n} D_{k} \cdot \overline{D_{k}}$ is a nonnegative function on $M$, Sca is the scalar curvature of $M$ and $\widetilde{R i c}_{a \bar{\beta}}$ is the Ricci tensor of $N$. For the the proof of Theorem 2.4 one can refer to [3] (or Corollary 4.4 in [12]) for details.

\section{Proofs of Theorem 1.2}

In this section we will prove Theorem 1.2 .

Let $M$ be any finite cover of the moduli space $\mathbb{M}_{g}$. If necessary we take a finite cover of $M$ again, still denoted by $M$, such that $M$ is a manifold. We lift the perturbed Ricci metric $\|\cdot\|_{L S Y}$ in Theorem 2.3 onto $M$. Let $\|\cdot\|$ be a complete finite-volume Hermitian metric on $M$. We consider the identity map

$$
i:(M,\|\cdot\|) \rightarrow\left(M,\|\cdot\|_{L S Y}\right) .
$$

It is clear that $i$ is holomorphic. We let $v$ be the ratio of intermediate volume elements for $i$ as in equation (2.3).

Lemma 3.1. For any $p \in(M,\|\cdot\|)$,

$$
v(p)>0 .
$$

Proof. For any $p \in(M,\|\cdot\|), v(p)>0$ follows from the fact that $i:(M, \| \cdot$ $\|) \rightarrow\left(M,\|\cdot\|_{L S Y}\right)$ is biholomorphic.

Now we are ready to prove Theorem 1.2 
Proof of Theorem 1.2. Assume that $\inf _{p \in(M,\|\cdot\|)} \mathrm{Sca}(p) \geqslant 0$. We will argue it by getting a contradiction. Let $\Delta$ be the Laplace operator on $(M,\|\cdot\|)$.

Claim 1: $\Delta v(q)>0$ for any $q \in(M,\|\cdot\|)$.

Proof of Claim 1. First by Theorem 2.3 we know that there exists a constant $C_{2}>0$ such that

$$
R i c_{\|\cdot\|_{L S Y}} \leqslant-C_{2}<0
$$

From Lemma 3.1 we know that $v>0$. Since $\inf _{p \in(M,\|\cdot\|)} \operatorname{Sca}(p) \geqslant 0$, by Theorem 2.4 we have

$$
\Delta v \geqslant-2 v\left(\sum_{1 \leqslant \alpha, \beta, k \leqslant n} a_{\alpha k} \bar{a}_{\beta k} \widetilde{R i c}_{a \bar{\beta}}\right) .
$$

Since $v>0$, inequalities (3.1) and (3.2) lead to

$$
\begin{aligned}
\Delta v & \geqslant 2 C_{2} v \cdot\left(\sum_{1 \leqslant \alpha, k \leqslant n}\left|a_{\alpha k}\right|^{2}\right) \\
& \geqslant 2 C_{2}(3 g-3) v^{\frac{3 g-2}{3 g-3}} \quad \text { (by the AM-GM inequality) } \\
& >0 .
\end{aligned}
$$

The remaining argument is inspired by the proof of Theorem 9.1 in 2 and section 8.12 in 14 . We remark that it is not known that $v$ is bounded from above, so the following Claim (2) can not directly follow from the results in [19].

Claim 2: $v$ is a constant on $(M,\|\cdot\|)$.

Proof of Claim 2. Let $g_{t}$ denote the flow generated by the vector field $\nabla v$. Since $(M,\|\cdot\|)$ is complete, $g_{t}$ is defined for all $t \geqslant 0$.

Assume that $v$ is not a constant and let $p_{0} \in M$ such that $\nabla v\left(p_{0}\right) \neq 0$. Along the flow line of $g_{t}$ starting at $p_{0}, v$ is increasing since for all $s_{2}>s_{1} \geqslant$ 0 ,

$$
v\left(g_{s_{2}}\left(p_{0}\right)\right)-v\left(g_{s_{1}}\left(p_{0}\right)\right)=\int_{s_{1}}^{s_{2}}\left\|\nabla v\left(g_{t}\left(p_{0}\right)\right)\right\| d t \geqslant 0
$$

That is

$$
v\left(g_{s_{2}}\left(p_{0}\right)\right) \geqslant v\left(g_{s_{1}}\left(p_{0}\right)\right) \quad \forall s_{2}>s_{1} \geqslant 0 .
$$

Since we assume that $\nabla v\left(p_{0}\right) \neq 0$, let $s_{2}=1$ and $s_{1}=0$ we have

$$
v\left(g_{1}\left(p_{0}\right)\right)>v\left(p_{0}\right)>0 .
$$


Therefore there exists a small enough constant $r_{0}>0$ such that

$$
\inf _{q \in B\left(p_{0}, r_{0}\right)} v\left(g_{1}(q)\right)>\sup _{q \in B\left(p_{0}, r_{0}\right)} v(q)
$$

where $B\left(p_{0}, r_{0}\right)$ is the geodesic ball centered at $p_{0}$ of radius $r_{0}$.

In particular we have

$$
B\left(p_{0}, r_{0}\right) \cap g_{1}\left(B\left(p_{0}, r_{0}\right)\right)=\emptyset .
$$

Inequality (3.6) and equation (4.8) give that

$$
B\left(p_{0}, r_{0}\right) \cap g_{n}\left(B\left(p_{0}, r_{0}\right)\right)=\emptyset \quad \forall n \in \mathbb{Z}^{+} .
$$

Which also implies

$$
g_{n}\left(B\left(p_{0}, r_{0}\right)\right) \cap g_{m}\left(B\left(p_{0}, r_{0}\right)\right)=\emptyset \quad \forall n \neq m \in \mathbb{Z}^{+} .
$$

Otherwise there exist two positive integers $n_{0}>m_{0} \geqslant 1$ and $q_{1}, q_{2} \in$ $B\left(p_{0}, r_{0}\right)$ such that $g_{n_{0}}\left(q_{1}\right)=g_{m_{0}}\left(q_{2}\right)$. Since $g_{t}$ is a flow, $g_{n_{0}-m_{0}}\left(q_{1}\right)=q_{2}$ which contradicts equation (3.11).

On the other hand, for any $t_{0}>0$ (we use Proposition 18.18 in 9]), we have

$$
\begin{aligned}
\left.\frac{d \operatorname{Vol}\left(g_{t}\left(B\left(p_{0}, r_{0}\right)\right)\right)}{d t}\right|_{t=t_{0}} & =\left.\int_{B\left(p_{0}, r_{0}\right)} \frac{d}{d t}\right|_{t=t_{0}} g_{t}^{*}(\mathrm{dVol}) \\
& =\int_{B\left(p_{0}, r_{0}\right)} g_{t_{0}}^{*}\left(\mathrm{E}_{\nabla v}(\mathrm{dVol})\right) \\
& =\int_{B\left(p_{0}, r_{0}\right)} g_{t_{0}}^{*}(\operatorname{div}(\nabla(v)) \mathrm{dVol}) \\
& =\int_{g_{t_{0}}\left(B\left(p_{0}, r_{0}\right)\right)} \Delta v \mathrm{dVol}
\end{aligned}
$$

From Claim (1) we have

$$
\left.\frac{d \operatorname{Vol}\left(g_{t}\left(B\left(p_{0}, r_{0}\right)\right)\right)}{d t}\right|_{t=t_{0}}>0 \quad \forall t_{0}>0 .
$$

That is the flow $g_{t}$ is volume increasing.

Thus, equation (3.12) and inequality (3.17) give that

$$
\begin{aligned}
\operatorname{Vol}((M,\|\cdot\|)) & \geqslant \operatorname{Vol}\left(\cup_{k=1}^{\infty} g_{k}\left(B\left(p_{0}, r_{0}\right)\right)\right) \\
& =\sum_{k=1}^{\infty} \operatorname{Vol}\left(g_{k}\left(B\left(p_{0}, r_{0}\right)\right)\right) \\
& \geqslant \sum_{k=1}^{\infty} \operatorname{Vol}\left(B\left(p_{0}, r_{0}\right)\right) \\
& =\infty
\end{aligned}
$$

which contradicts our assumption that $(M,\|\cdot\|)$ has finite volume. 
It is clear that Claim (1) and Claim (2) can not simultaneously hold. Therefore, the proof is completed.

\section{Proofs of Theorem 1.3}

As the same in section 3 we let $M$ be any finite cover of the moduli space $\mathbb{M}_{g}$ and $\|\cdot\|_{L S Y}$ be the perturbed Ricci metric in Theorem 2.3. Let $k_{1}, k_{2}$ be two positive constants and $\|\cdot\|$ be a Hermitian metric on $M$ with

$$
k_{1}\|\cdot\| \leqslant\|\cdot\|_{T} \leqslant k_{2}\|\cdot\| \text {. }
$$

From Theorem 2.1 and Theorem 2.2, up to some uniform constants, we may assume that

$$
k_{1}\|\cdot\| \leqslant\|\cdot\|_{L S Y} \leqslant k_{2}\|\cdot\| .
$$

Use the same notations in section 3 we let $v$ be the ratio of intermediate volume elements for $i$ as in equation (2.3).

Lemma 4.1. For any $p \in(M,\|\cdot\|)$, we have

$$
v(p) \geqslant k_{1}^{6 g-6}>0
$$

In particular, $(M,\|\cdot\|)$ has finite volume.

Proof. Since $k_{1}\|\cdot\| \leqslant\|\cdot\|_{L S Y}$, linear algebra gives that

$$
k_{1}^{6 g-6} \mathrm{dVol}_{\|\cdot\|} \leqslant \mathrm{dVol}_{\|\cdot\|_{L S Y}} .
$$

The conclusion follows from equation (2.4) and inequality (4.2).

Since $\left(M,\|\cdot\|_{L S Y}\right)$ has finite volume, inequality (4.2) tells that $(M,\|\cdot\|)$ also has finite volume.

Lemma 4.2. $(M,\|\cdot\|)$ is complete.

Proof. It directly follows from our assumption that $\|\cdot\|_{T} \leqslant k_{2}\|\cdot\|$ and the fact that the Teichmüller metric is complete.

Now we are ready to prove Theorem 1.3.

Proof of Theorem 1.3. Theorem 2.3 tells that there exists a constant $C_{2}>0$ such that

$$
R i c_{\|\cdot\|_{L S Y}} \leqslant-C_{2}<0 .
$$

We let $k_{3}:=\inf _{p \in(M,\|\cdot\|)} \operatorname{Sca}(p)$. From Lemma 3.1 we know that $v>0$. Thus, from Theorem 2.4 we have

$$
\begin{aligned}
\Delta v & \geqslant 2 v \cdot k_{3}-2 v\left(\sum_{1 \leqslant \alpha, \beta, k \leqslant n} a_{\alpha k} \bar{a}_{\beta k} \widetilde{R i c}_{a \bar{\beta}}\right) \\
& \geqslant 2 v\left(k_{3}+C_{2}(3 g-3) v^{\frac{1}{3 g-3}}\right) \quad \text { (by the AM-GM inequality). }
\end{aligned}
$$

From Lemma 4.1 we have 


$$
\Delta v \geqslant 2 k_{1}^{6 g-6} \cdot\left(k_{3}+C_{2}(3 g-3) k_{1}^{2}\right) .
$$

We choose $K\left(k_{1}, g\right)=\frac{C_{2}(3 g-3) k_{1}^{2}}{2}>0$.

Claim : $\inf _{p \in(M,\|\cdot\|)} \operatorname{Sca}(p) \leqslant-K\left(k_{1}, g\right)<0$.

Proof of Claim. Assume it is not. That is

$$
\inf _{p \in(M,\|\cdot\|)} \operatorname{Sca}(p) \geqslant-K\left(k_{1}, g\right) .
$$

Inequalities (4.4) and (4.5) lead to

$$
\Delta v \geqslant C_{2} \cdot(3 g-3) \cdot k_{1}^{6 g-4}>0 .
$$

There are more information coming from the conditions of Theorem 1.3. We provide two different methods to finish the proof of the claim.

Method (1). Since $(M,\|\cdot\|)$ is complete and has finite volume, it follows from inequality (4.6) and the same argument as in the proof of Claim (2) in section 3 that $v$ is a constant which contradicts inequality (4.6).

Method (2). First from the right side of inequality (4.1) and by using a same argument in the proof of Lemma 4.1 there exists a positive constant $C_{3}$ such that

$$
\sup _{p \in(M,\|\cdot\|)} v(p) \leqslant C_{3} .
$$

Fix $p_{0} \in M$ and let $B\left(p_{0}, r\right)$ be the closed geodesic ball of $(M,\|\cdot\|)$ centered at $p_{0}$ of radius $r$. Then for any $t>0$ there exists a bump function $f(x):(M,\|\cdot\|) \rightarrow[0, \infty)$ which is a Lipschitz continuous function and a constant $C_{4}>0$ such that

(i). $f \equiv 1$ on $B\left(p_{0}, t\right)$ and $f \equiv 0$ on $M-B\left(p_{0}, 2 t\right)$.

(ii). $\|\nabla f\| \leqslant \frac{C_{4}}{t}$ a.e. on $M$.

(For the existence of such bump functions one can refer to [19]).

First since $v>0$ and $\Delta v>0$ we have

$$
\Delta v^{2} \geqslant 2\|\nabla v\|^{2} \text {. }
$$

Let $<,>$ be the Riemannian inner product associated to the metric $\|\cdot\|$. The Stokes' theorem and inequality (4.8) give

$$
\begin{aligned}
0 & =\int_{B\left(p_{0}, 2 t\right)} \operatorname{div}\left(f^{2} \nabla\left(v^{2}\right)\right) \\
& \geqslant 4 \int_{B\left(p_{0}, 2 t\right)} f \cdot v \cdot\langle\nabla f, \nabla v\rangle+2 \int_{B\left(p_{0}, 2 t\right)} f^{2} \cdot\|\nabla v\|^{2} .
\end{aligned}
$$

The Cauchy-Schwarz inequality leads to 


$$
\begin{aligned}
\int_{B\left(p_{0}, 2 t\right)} f^{2} \cdot\|\nabla v\|^{2} & \leqslant-2 \int_{B\left(p_{0}, 2 t\right)} f \cdot v \cdot\langle\nabla f, \nabla v> \\
& \leqslant 2 \sqrt{\int_{B\left(p_{0}, 2 t\right)} f^{2} \cdot\|\nabla v\|^{2}} \cdot \sqrt{\int_{B\left(p_{0}, 2 t\right)} v^{2} \cdot\|\nabla f\|^{2}} .
\end{aligned}
$$

That is

$$
\int_{B\left(p_{0}, 2 t\right)} f^{2} \cdot\|\nabla v\|^{2} \leqslant 4 \int_{B\left(p_{0}, 2 t\right)} v^{2} \cdot\|\nabla f\|^{2} .
$$

Since $f \equiv 1$ on $B\left(p_{0}, t\right)$ and $\|\nabla f\| \leqslant \frac{C_{4}}{t}$ a.e. on $M$, we replace inequality (4.9) by

$$
\int_{B\left(p_{0}, t\right)}\|\nabla v\|^{2} \leqslant \frac{4 C_{4}^{2}}{t^{2}} \int_{B\left(p_{0}, 2 t\right)} v^{2} .
$$

By inequality (4.7),

$$
\int_{B\left(p_{0}, t\right)}\|\nabla v\|^{2} \leqslant \frac{4 C_{3}^{2} C_{4}^{2}}{t^{2}} \operatorname{Vol}\left(B\left(p_{0}, 2 t\right)\right) .
$$

Since we assume that $(M,\|\cdot\|)$ has finite volume, there exists a constant $C_{5}>0$ such that

$$
\int_{B\left(p_{0}, t\right)}\|\nabla v\|^{2} \leqslant \frac{C_{5}}{t^{2}}
$$

Since $(M,\|\cdot\|)$ is complete and open, we let $t \rightarrow \infty$, it follows from inequality (4.12) that $\nabla v \equiv 0$ on $M$. That is, $v$ is a constant on $M$ which contradicts inequality (4.6).

For the second method above, we use the same argument as in [19].

It is clear that the conclusion follows from the claim.

\section{Proof of Theorem 1.4}

Recall a Hermitian manifold $(M,<,>)$ is almost if there exists an almost complete structure $\mathbb{J}$ on $M$ such that

$$
<\mathbb{J} \circ V, \mathbb{J} \circ W>=<V, W>
$$

for all tangent vectors $V$ and $W$. It is clear that a Kähler manifold is almost Hermitian.

If we assume that both $M$ and $N$ in subsection 2.3 are almost Hermitian, Goldberg and Harél in [8] proved that the term $\sum_{k=1}^{n} D_{k} \cdot \overline{D_{k}}$ in Chern's 
formula (see Theorem 2.4) satisfies

$$
\sum_{k=1}^{n} D_{k} \cdot \overline{D_{k}}=\frac{\|\nabla v\|^{2}}{4 v}
$$

Thus, a direct computation for Theorem 2.4 gives that

$$
\Delta \log (v)=2 \cdot\left(\mathrm{Sca}-\sum_{1 \leqslant \alpha, \beta, k \leqslant n} a_{\alpha k} \bar{a}_{\beta k} \widetilde{R i c} \overline{a \bar{\beta}}\right) .
$$

(One can see formula (10) in [8] for details.)

Before we prove Theorem [1.4 let us recall a theorem of S. T. Yau (see Theorem 1 in [19]) which is crucial for this section.

Let $N$ be a complete Riemannian manifold and $\Delta$ be the Laplace operator of $N$. Assume that $u$ and $h$ are two functions on $M$ satisfying the following equation

$$
\Delta \log u=h .
$$

Then the following theorem says that

Theorem 5.1 (Yau). Suppose $h$ is bounded from below by a constant and $0<\int_{N} h(p) \mathrm{d} \operatorname{Vol}(p) \leqslant \infty$. Then $\int_{N} u^{n}(p) \mathrm{d} \operatorname{Vol}(p)=\infty$ for $n>0$, unless $u$ is a constant function.

Let $M$ be a finite cover of the moduli space and $|\cdot| \mid$ be an almost Hermitian metric with $\|\cdot\| \approx\|\cdot\|_{T}$. We use the same notations here as in section 3 , In our setting we let

$$
N=(M,\|\cdot\|), \quad u=v
$$

and

$$
h=2 \cdot\left(\mathrm{Sca}-\sum_{1 \leqslant \alpha, \beta, k \leqslant(3 g-3)} a_{\alpha k} \bar{a}_{\beta k} \widetilde{R i c} \overline{a \beta}\right) .
$$

Since the perturbed Ricci metric $\|\cdot\|_{L S Y}$ is Kähler and $(M,\|\cdot\|)$ is almost Hermitian, formula (5.1) exactly tells us that

$$
\Delta \log v=h .
$$

Now we are ready to prove Theorem 1.4 .

Proof of Theorem 1.4. First since $\|\cdot\| \approx\|\cdot\|_{T}$, From Theorem 2.1 and Theorem 2.2, we may assume that

$$
k_{1}\|\cdot\| \leqslant\|\cdot\|_{L S Y} \leqslant k_{2}\|\cdot\| .
$$

The proof of Lemma 4.1 gives that

$$
k_{1}^{6 g-6} \leqslant v \leqslant k_{2}^{6 g-6} .
$$

By using a similar argument in the previous proofs, from Theorem 2.3 we have, for any $p \in(M,\|\cdot\|)$, 


$$
\begin{aligned}
h(p) & =2 \cdot\left(\operatorname{Sca}(p)-\sum_{1 \leqslant \alpha, \beta, k \leqslant(3 g-3)} a_{\alpha k}(p) \bar{a}_{\beta k}(p) \widetilde{R i c}_{a \bar{\beta}}(p)\right) \\
& \geqslant 2 \cdot \inf _{p \in(M,\|\cdot\|))} \operatorname{Sca}(p)+2 C_{2}\left(\sum_{1 \leqslant \alpha, \beta \leqslant(3 g-3)}\left|a_{\alpha \beta}(p)\right|^{2}\right) \\
& \geqslant 2 \cdot \inf _{p \in(M,\|\cdot\|))} \operatorname{Sca}(p)+2 C_{2}(3 g-3) v^{\frac{1}{3 g-3}} \quad \text { (by the AM-GM inequality) } \\
& >2 \cdot \inf _{p \in(M,\|\cdot\| \mid \|))} \operatorname{Sca}(p) \\
& >-\infty
\end{aligned}
$$

where we apply the assumption that the scalar curvature of $(M,\|\cdot\|)$ is bounded from below for the last step. That is, $h$ is bounded from below on $(M,\|\cdot\|)$.

We finish the proof through the following two cases.

Case (1). $v$ is a constant on $(M,\|\cdot\|)$.

If $v$ is a constant, by equation (5.3) we have $h \equiv 0$. That is,

$$
\mathrm{Sca}=\sum_{1 \leqslant \alpha, \beta, k \leqslant n} a_{\alpha k} \bar{a}_{\beta k} \widetilde{\operatorname{Ric}}_{a \bar{\beta}} .
$$

It follows from Theorem 2.3 and equation (5.6) that

$$
\operatorname{Sca}(p)<0 \quad \forall p \in(M,\|\cdot\|) .
$$

It is clear that the total mass

$$
\int_{p \in(M,\|\cdot\|)} \operatorname{Sca}(p) \mathrm{dVol}(p)<0 .
$$

Case (2). $v$ is not a constant on $(M,\|\cdot\|)$.

Since $\left(M,\|\cdot\|_{L S Y}\right)$ has finite volume, from inequality (5.5) we have

$$
\int_{p \in(M,\|\cdot\|)} v^{n}(p) \mathrm{d} \operatorname{Vol}(p)<\infty \quad \forall n>0 .
$$

We already show that $h$ is bounded from below on $(M,\|\cdot\|)$. Thus, from Theorem 5.1 we know that

$$
\int_{p \in(M,\|\cdot \cdot\|)} h(p) \mathrm{d} \operatorname{Vol}(p) \leqslant 0 .
$$

That is, 


$$
\int_{p \in(M,\|\cdot\|)} \operatorname{Sca}(p) \mathrm{d} \operatorname{Vol}(p) \leqslant \int_{p \in(M,\|\cdot\|)} \sum_{1 \leqslant \alpha, \beta, k \leqslant(3 g-3)} a_{\alpha k}(p) \bar{a}_{\beta k}(p) \widetilde{\operatorname{Ric}} \overline{a \bar{\beta}}(p) \mathrm{dVol}(p) .
$$

By Theorem 2.3 and the AM-GM inequality we have

$$
\begin{aligned}
\int_{p \in(M,\|\cdot\|)} \operatorname{Sca}(p) \mathrm{d} \operatorname{Vol}(p) & \leqslant-\int_{p \in(M,\|\cdot\|)} C_{2}\left(\sum_{1 \leqslant \alpha, \beta \leqslant(3 g-3)}\left|a_{\alpha \beta}(p)\right|^{2}\right) \mathrm{d} \operatorname{Vol}(p) \\
& \leqslant-\int_{p \in(M,\|\cdot\|)} C_{2}(3 g-3) v(p)^{\frac{1}{3 g-3}} \mathrm{dVol}(p) \\
& <0 .
\end{aligned}
$$

Where we apply the fact that $v>0$ for the last step.

\section{ACKNOWLEDGEMENT}

The author would like to thank Kefeng Liu, Michael Wolf and Scott Wolpert for their interests. He deeply thank Benson Farb for his encouragement for writing this article. This work was partially completed during a reading seminar at Rice university, organized by Michael Wolf and Robert Hardt. The author would like to thanks for their consistent help. He also would like to acknowledge support from U.S. National Science Foundation grants DMS 1107452, 1107263, 1107367 "RNMS: Geometric structures And Representation varieties"(the GEAR Network).

\section{REFERENCES}

[1] Lars V. Ahlfors, Some remarks on Teichmüller space of Riemann surfaces, Ann. of Math. 74 (1961), no. 2, 171-191.

[2] R. Canary, Ends of hyperbolic 3-manifolds, J. Amer. Math. Soc. 6(1993), 1-35.

[3] S. S. Chern, On holomorphic mappings of hermitian manifolds of the same dimension, Entire functions and related parts of analysis, American Mathematical Society, Providence, R. I., 1968, 157-170.

[4] T. Chu, The Weil-Petersson metric in the moduli space, Chinese J. Math. 4 (1976), no. 2, 29-51.

[5] B. Farb, Some problems on mapping class groups and moduli space, Problems on Mapping Class Groups and Related Topics, Proc. Sympos. Pure Math., vol. 74, Amer. Math. Soc., Providence, R. I., 2006, pp. 11-55.

[6] B. Farb, private communication.

[7] B. Farb and S. Weinberger, Positive scalar curvature metrics on the moduli space of Riemann surfaces, in preparation.

[8] S. I. Goldberg and Z. Harél, Mappings of almost hermitian manifolds, J. Differential Geom. 14 (1979), 67-80.

[9] J. M. Lee, Introduction to smooth manifolds, Springer, 2003.

[10] K. Liu, X. Sun and S. T. Yau, Canonical metrics on the moduli space of Riemann surfaces, I. J. Differential Geom. 68 (2004), no. 3, 571-637.

[11] K. Liu, X. Sun and S. T. Yau, Canonical metrics on the moduli space of Riemann surfaces, II. J. Differential Geom. 69 (2005), no. 1, 163-216. 
[12] Y. Lu, Holomorphic mappings of complex manifolds, J. Differential Geom. 2 (1968), 299-312.

[13] C. McMullen, The moduli space of Riemann surfaces is Kähler hyperbolic, Ann. of Math. (2) 151 (2000), no. 1, 327-357.

[14] W. Thurston, The geometry and topology of 3-manifolds, Lecture notes, Princeton Univ.

[15] A. J. Tromba, On a natural algebraic affine connection on the space of almost complex structures and the curvature of Teichmüller space with respect to its Weil-Petersson metric, Manuscripta Math. 56 (1986), no. 4, 475-497.

[16] S. Trapani, On the determinant of the bundle of meromorphic quadratic differentials on the Deligne-Mumford compactification of the moduli space of Riemann surfaces, Math. Ann. 293 (1992), no. 4, 681-705.

[17] S. Wolpert, Noncompleteness of the Weil-Petersson metric for Teichmüller space, Pacific J. Math. 61 (1975), no. 2, 573-577.

[18] S. Wolpert, Chern forms and the Riemann tensor for the moduli space of curves, Invent. Math. 85 (1986), no. 1, 119-145.

[19] S. T. Yau, Some function theoretic properties of complete Riemannian manifolds and their applications to geometry, Indiana Univ. Math. J. 25 (1976), 659-670.

[20] S. T. Yau, A general Schwarz lemma for Kähler manifolds, Amer. J. Math. 100 (1978), no. 1, 197-203.

[21] S. K. Yeung, Quasi-isometry of metrics on Teichmüller spaces, Int. Math. Res. Not. (2005), no. 4, 239-255.

Department of Mathematics, Rice University, Houston, Texas, 77005-1892,

E-mail address: yw22@rice.edu 